\title{
Eficiencia del análisis gasométrico arterial y venoso como predictor de la mortalidad en pacientes con choque séptico: estudio de cohorte prolectivo
}

Efficiency of blood gas analysis as mortality predictor on septic shock patients: prolective cohort study Eficiência da gasometria arterial e venosa como preditor de mortalidade em pacientes em choque séptico: estudo de coorte prolectivo

\author{
Óscar Emilio Palacios-Calderón, ${ }^{*}$ Jorge Eliel Monter-Vigueras, ${ }^{\ddagger}$ Janet Silvia Aguirre-Sánchez, ${ }^{\S}$ \\ Juvenal Franco-Granillo,"l Gilberto Camarena-Alejo," Alfredo Aisa-Álvarez ${ }^{* *}$
}

\section{RESUMEN}

Introducción: El análisis gasométrico es probablemente la herramienta de valoración más utilizada en la Unidad de Terapia Intensiva. Una adecuada comprensión y uso del muestreo arterial y venoso central/arteria pulmonar hace posible interpretar la mayoría de alteraciones respiratorias, circulatorias y metabólicas, las cuales pueden presentarse en el paciente con choque séptico.

Objetivo: Evaluar la eficiencia de un análisis gasométrico arterial y venoso para predecir la mortalidad de pacientes con choque séptico.

Material y métodos: Estudio de cohorte prolectivo que se realizó en dos unidades de terapia intensiva de adultos desde enero 2018 a julio 2019. Se incluyeron a pacientes adultos mayores de 18 años con diagnóstico de choque séptico según los criterios SEPSIS-3. Todo paciente ingresado con diagnóstico de choque séptico le fue realizado un análisis gasométrico arterial y venoso al mismo tiempo para la realización de los siguientes cálculos: $\mathrm{PaO}_{2} / \mathrm{FiO}_{2}, \mathrm{ScvO}_{2}$, Qs/Qt, Dv-aCO, Da-vO, Dv-aCO $/ \mathrm{Da}_{2} \mathrm{vO}_{2}, \mathrm{IEO}_{2}$.

Resultados: Se analizaron 136 pacientes. La edad promedio fue de 66 años. IMC media de $25.17 \mathrm{~kg} / \mathrm{m}^{2}$, con 74 hombres (54\%) dentro de las comorbilidades más frecuentes: tabaquismo, hipertensión, cáncer y diabetes. En $75 \%$ de los ingresos, éstos fueron no quirúrgicos, siendo el motivo respiratorio $(30 \%)$ el más frecuente; $30 \%$ de los pacientes falleció. El antecedente de enfermedad renal crónica tuvo una relación estadísticamente significativa con la mortalidad: RR de 3.73 (IC 95\% 1.28-10.8); $p=0.01$. El taller gasométrico mostró la $\mathrm{Da}^{-\mathrm{vO}} \mathrm{O}_{2}$ con una media de $2.91 \mathrm{~mL} / \mathrm{dL}$ (hiperdinamia), la cual tuvo una relación directa con la mortalidad: RR de 0.58 (IC 95\% 0.39-0.87); $p=0.008$; asimismo, el índice Dv-aCO $/ \mathrm{Da}^{\mathrm{v}} \mathrm{O}_{2}$ tuvo una $\mathrm{p}=0.023$.

Conclusión: El taller gasométrico se considera una herramienta útil y no invasiva para el análisis de los distintos cambios fisiopatológicos en el paciente con choque séptico. Un parámetro del taller gasométrico aislado normal o alterado no valora el aporte de oxígeno tisular $\left(\mathrm{DO}_{2}\right)$. Considerando los efectos deletéreos de la sobreestimulación generada por la actividad simpática y el hallazgo de un perfil de hiperdinamia mayormente asociado con mortalidad, la terapéutica con betabloqueadores puede ser un tratamiento atractivo, el cual se dirija a pacientes con choque séptico.

Palabras clave: Taller gasométrico, choque séptico, hiperdinamia.

\section{ABSTRACT}

Introduction: Blood gas analysis is probably the most common diagnostic tool used in intensive care. A proper understanding and use of arterial and pulmonary/central venous blood gas analysis makes it possible to correctly interpret most of the respiratory, circulatory and metabolic derangements which may occur in septic shock patients.

\footnotetext{
* Medicina Interna. Medicina Crítica. Departamento de Medicina Crítica, Centro Mé dico ABC.

¥ Medicina de Urgencias. Medicina Crítica. Departamento de Medicina Crítica, Centro Médico ABC.

$\S$ Medicina Interna y Medicina Crítica. Dirección y Administración de Instituciones de Salud. Subjefe del Departamento de Medicina Crítica, Centro Médico ABC. Campus Observatorio. Ciudad de México, México.

" Medicina Interna y Medicina Crítica. División de Medicina Crítica del Centro Médico ABC. Ciudad de México, México.

『 Cardiología y Medicina Crítica. Dirección y Administración De Instituciones de Salud. Departamento de Medicina Crítica, Centro Médico ABC. Campus Santa Fe. Ciudad de México, México.

${ }^{* *}$ Medicina Interna y Medicina Crítica. Departamento de Medicina Crítica, Centro Médico ABC, Campus Santa Fe. Ciudad de México, México.
}

Recepción: 20/09/2019. Aceptación: 09/10/2019.

www.medigraphic.com/medicinacritica
Objective: To assess the effectiveness of arterial and venous blood analysis in predicting mortality of patients with septic shock.

Material and methods: Prolective cohort study conducted in two adult intensive care units, period January 2018-July 2019. Adult patients +18 years who entered the intensive therapy unit of $A B C$ Medical Center, both campuses, with septic shock diagnosis were included according to SEPSIS-3 criteria. For every patient admitted with a diagnosis of septic shock, arterial and venous blood analysis was performed at the same time for the following calculations: $\mathrm{PaO}_{2}$ $\mathrm{FiO}_{2}, \mathrm{ScvO}_{2}, \mathrm{Qs} / \mathrm{Qt}, \mathrm{Dv}-\mathrm{aCO}, \mathrm{Da}-\mathrm{VO}_{2}, \mathrm{Dv}-\mathrm{aCO} / \mathrm{Da}-\mathrm{VO}_{2}, \mathrm{IEO}_{2}$

Results: 136 patients were included. Average age was 66 years. Average BMI $25.17 \mathrm{~kg} / \mathrm{m}^{2}, 74$ (54\%) were men, most common comorbilities were smoking, hypertension, cancer and diabetes. $75 \%$ of income was non-surgical, with respiratory causes (30\%) as the most frequent medical conditions. $30 \%$ of patients died. Past history of chronic kidney disease was statistically significant with mortality OR $3.73(95 \% \mathrm{Cl} 1.28-10.8) ; p=0.01$. The blood gas analysis showed that $\mathrm{Da}-\mathrm{vO}_{2}$ with an average of $2.91 \mathrm{~mL} / \mathrm{dL}$ (hyperdynamics) was directly related to mortality OR 0.58 (95\% Cl 0.39-0.87); $p=0.008$; also, the $\mathrm{Dv}$-aCO$/ \mathrm{Da}-\mathrm{vO}_{2} \mathrm{p}=0.023$ index.

Conclusion: Blood gas analysis is a useful, non-invasive tool for the analysis of the different pathophysiological changes in the patient in septic shock. An isolated parameter does not indicate an adequate or inadequate tissue oxygen supply $\left(\mathrm{DO}_{2}\right)$. Considering the effects of overstimulation generated by sympathetic activity and the finding of a hyperdynamic profile mostly associated with mortality, therapeutic management with beta blockers may be an attractive treatment in selected septic shock patients.

Keywords: Blood gas analysis, septic shock, hyperdynamic profile.

\section{RESUMO}

Introdução: A análise gasométrica é provavelmente a ferramenta de avaliação mais utilizada na unidade de terapia intensiva. $O$ entendimento e uso adequados da amostragem arterial e venosa central/artéria pulmonar possibilitam a interpretação da maioria dos distúrbios respiratórios, circulatórios e metabólicos que podem ocorrer no paciente em choque séptico.

Objetivos: Avaliar a eficiência de uma análise gasométrica arterial e venosa na predição da mortalidade de pacientes com choque séptico.

Material e métodos: Estudo de coorte prolectivo realizado em duas unidades de terapia intensiva para adultos, no período de janeiro de 2018 a julho de 2019. Foram incluídos pacientes adultos acima de 18 anos com diagnóstico de choque séptico, de acordo com os critérios do SEPSIS-3. Todos os pacientes admitidos com diagnóstico de choque séptico foram submetidos à análise gasométrica arterial e venosa ao mesmo tempo para realizar os seguintes cálculos: $\mathrm{PaO}_{2} / \mathrm{FiO}_{2}, \mathrm{ScvO}_{2}, \mathrm{Qs} / \mathrm{Qt}, \mathrm{Dv}$-aCO$, \mathrm{Da}-\mathrm{CO}_{2}, \mathrm{Dv}$-aCO$/$ $\mathrm{Da}-\mathrm{VO}_{2}, \mathrm{IEO}_{2}$

Resultados: 136 pacientes foram analisados. Idade média de 66 anos. IMC médio de $25.17 \mathrm{~kg} / \mathrm{m}^{2}, 74$ (54\%) homens, nas comorbidades mais frequentes: tabagismo, hipertensão, câncer e diabetes. 75\% das internações não foram cirúrgicas, sendo a razão respiratória (30\%) a mais frequente. $30 \%$ dos pacientes morreram. A história de doença renal crônica teve relação estatisticamente significante com a mortalidade RR 3.73 (IC 95\% 1.28-10.8) $p$ $=0.01$. A análise gasométrica mostrou $\mathrm{Da}-\mathrm{vO}, \mathrm{com}$ uma média de $2.91 \mathrm{~mL} /$ $d L$ (hiperdinâmica) que teve relação direta com a mortalidade $R R 0.58$ (IC95\% 0.39-0.87) $p=0.008$; da mesma forma, o índice $\mathrm{Dv}-\mathrm{aCO} / \mathrm{Da}-\mathrm{vO}_{2} p=0.023$. Conclusão: $A$ análise gasométrica é considerada uma ferramenta útil e não invasiva para a análise das diferentes alterações fisiopatológicas do paciente em choque séptico. Um parâmetro da análise gasométrica isolada normal ou alterada não avalia a contribuição do oxigênio tecidual $\left(\mathrm{DO}_{2}\right)$. Considerando os efeitos deletérios da superestimulação gerada pela atividade simpática e o achado de um perfil hiperdinâmico associado principalmente à mortalidade, a terapia com betabloqueadores pode ser um tratamento atraente para pacientes em choque séptico.

Palavras-chave: Análise gasométrica, choque séptico, hiperdinâmica. 


\section{INTRODUCCIÓN}

La sepsis y el choque séptico son problemas de salud pública a nivel mundial, los cuales representan un alto costo para los sistemas de salud. Por un lado, sepsis se define como la disfunción orgánica que pone en riesgo la vida causada por una inadecuada respuesta del huésped ante una infección; ${ }^{1}$ por el otro, el choque séptico es un subconjunto de sepsis con disfunción celular y metabólica asociada con una alta mortalidad, la cual puede llegar desde 35 hasta $40 \% .^{2-4}$ La sepsis es la mayor causa de mortalidad en las $\mathrm{UCl}$ de todo el mundo, ya que los niveles que alcanza son hasta $80 \%$ en enfermos con falla orgánica múltiple (FOM) ${ }^{5}$

El análisis gasométrico es posiblemente la herramienta diagnóstica que más se utiliza en terapia intensiva. El muestreo gasométrico arterial y venoso permite el diagnóstico y monitoreo de distintas condiciones fisiopatológicas, sea de aquellas funciones metabólicas, circulatorias y respiratorias que ocurren en el paciente con choque séptico. ${ }^{6}$

Ante la diversidad de valoraciones clínicas, hemodinámicas y metabólicas, es importante determinar qué parámetros micro y macrocirculatorios evaluados a través del taller gasométrico guardan relación con la mortalidad en pacientes con choque séptico, tanto de manera individual como en conjunto.

\section{MATERIAL Y MÉTODOS}

\section{Diseño del estudio, lugar y selección de los participantes}

Se trata de un estudio de cohorte prolectivo, el cual se llevó a cabo en el Departamento de Terapia Intensiva de dos Unidades Médicas de Tercer Nivel en la Ciudad de México.

El Comité de Ética Institucional revisó y aprobó el presente estudio. Antes de incluir a los pacientes, se obtuvo el consentimiento informado por escrito de cada uno de ellos o de sus responsables legales cuando fuera pertinente.

Basados en los criterios SEPSIS-3, se incluyeron a 136 pacientes mayores de 18 años con diagnóstico de choque séptico, quienes ingresaron a ambos Departamentos de Terapia Intensiva durante el periodo de enero de 2018 a julio de 2019. Se excluyeron a aquellos pacientes en quienes no se les pudiera realizar el muestreo de gases arteriales y aquellos pacientes con formato de voluntad anticipada; también, se excluyeron a pacientes embarazadas.

\section{Recolección de datos}

Todos los pacientes que ingresaron a la Unidad de Terapia Intensiva fueron evaluados para la obtención de las características demográficas, como edad, género, peso, talla, índice de masa corporal (IMC), enfermedades previas, ingesta de medicamentos y terapia de sustitución renal. También se anotó el registro del día de ingreso para hacer el cálculo de días de estancia hospitalaria.

Además, se calcularon las escalas pronósticas de APACHE II y SAPS II al ingreso a la Unidad de Terapia Intensiva, así como el puntaje de SOFA para disfunción orgánica (neurológico, respiratorio, hemodinámico, hepático y hematológico).

Todos los pacientes que ingresaron con diagnóstico de choque séptico, se les realizaron análisis gasométrico arterial y venoso central/arteria pulmonar al mismo tiempo para la obtención de los siguientes cálculos: $\mathrm{PaO}_{2} / \mathrm{FiO}_{2}, \mathrm{ScvO}_{2}, \mathrm{Qs} / \mathrm{Qt}, \mathrm{Dv}-\mathrm{aCO}_{2}, \mathrm{Da}-\mathrm{vO}_{2}, \mathrm{Dv}-\mathrm{aCO}_{2} /$ $\mathrm{Da}-\mathrm{vO}_{2}$ y $\mathrm{IEO}_{2}$.

\section{Definición de desenlaces}

El desenlace primario fue la eficiencia de las variables del taller gasométrico como determinantes individuales o grupales de mortalidad en pacientes con choque séptico.

El desenlace secundario fue el análisis de las variables del taller gasométrico entre sí y su relación con los días de estancia hospitalaria, así como los días de ventilación mecánica.

\section{Análisis estadístico}

La descripción de las variables cualitativas se llevó a cabo como mediana (con mínimo y máximo) o media (con desviación estándar), según fuera el caso; las variables cuantitativas se reportaron como frecuencias y porcentajes.

Para la evaluación comparativa de variables cuantitativas, se utilizó t de Student o U de Mann-Whitney, lo cual dependió de la evaluación previa de normalidad mediante Shapiro-Wilks o Kolmogórov-Smirnov. Para las variables cualitativas, se utilizó $\chi^{2}$ o prueba exacta de Fisher (si en la evaluación previa del valor esperado se encontró o no un valor $\leq 5$ ).

Se estimaron intervalos de confianza a 95\%, tanto para proporciones como para razones de momios. Para el nivel de significancia, se determinó que un valor de $p$ menor o igual a 0.05 se consideraría estadísticamente significativo.

El análisis multivariado se llevó a cabo con un análisis de regresión logística binaria para evaluar posibles confusores. La adecuación del modelo fue evaluada mediante la prueba de Hosmer-Lemeshow, la cual se consideró como relevante si la $p$ fue $>0.2$. Para el análisis estadístico, se utilizó el programa STATA versión 15. 


\section{Cálculo de muestra}

Fijando un nivel de significación $\alpha=0.05$, una potencia $1-\beta=0.80$, una desviación estándar $\sigma=0.5$, un coeficiente de determinación $\mathrm{R} 2=0$, una probabilidad de ocurrencia del evento de interés $\mathrm{pE}=0.30 \mathrm{y}$ el coeficiente de la variable principal del estudio $\gamma 1=1$, el tamaño de muestra se obtiene mediante la siguiente fórmula:

$$
\begin{gathered}
E=\frac{(1.9599+0.84)^{2}}{\left(0.5^{2}\right)\left(1^{2}\right)(1-0)}=32 \\
n=\frac{E}{p_{E}}=\frac{32}{0.30}=105
\end{gathered}
$$

Si consideramos para la regresión la fórmula de Freeman $\left[n=10^{*}(k+1)\right]$, tomando en cuenta cuatro variables independientes para el modelo con una $\mathrm{pE}$ de 0.30 , obtenemos el siguiente tamaño de muestra:

$$
\begin{gathered}
n=10^{\star}(4)=40 \\
n=40 / 0.30=133 \text { sujetos }
\end{gathered}
$$

Finalmente, el tamaño de muestra requerido, de acuerdo con la fórmula de Schoenfeld, fue de 105 pacientes. No obstante, se buscará incluir 133 pacientes, considerando pérdidas y censuras.

\section{RESULTADOS}

Se analizaron un total de 136 pacientes, generando un tamaño de muestra significativo para el estudio. En la Tabla 1, se presentan las características basales de los pacientes. La edad promedio del estudio fue de 66 años, con IMC con una media de $25.17 \mathrm{~kg} / \mathrm{m}^{2}$, lo que cataloga a la población en estudio en un grado de sobrepeso; de éstos, 74 pacientes (54\%) son hombres dentro de las comorbilidades más frecuentes: tabaquismo, hipertensión, cáncer y diabetes. De la cantidad de ingresos, $75 \%$ fue no quirúrgicos, siendo el motivo respiratorio $(30 \%)$ el más frecuente para ingreso a terapia intensiva. El riesgo nutricional de los pacientes fue bajo 4 (1-8). Los puntajes de severidad encontrados, APACHE II (16.69 \pm 7.74$)$, SAPS II (39.88 \pm 16.09$)$, SOFA $8(1-17)$, representaron un riesgo de mortalidad, el cual iba de 20 a $35 \%$; de este porcentaje, $30 \%$ de los pacientes falleció.

En la Tabla 2, encontramos que la Da-vO 2 (2.91 [1.34-7.7] vs 4.09 [1.1-8.7]; $p=0.024)$, es un factor estadísticamente significativo que se asocia con mortalidad, demostrando que el estado de hiperdinamia fue más prevalente en los no sobrevivientes (2.91 [1.347.7]). Asimismo, se encontró una diferencia estadística con el índice $\mathrm{Dv}-\mathrm{aCO}_{2} / \mathrm{Da}-\mathrm{vO}_{2}(2.06$ [0.5-6.66] vs 1.73 [0.23-9.24]; $p=0.023)$. El resto de análisis del taller gasométrico no tuvo ninguna diferencia significativa.

Al realizar el modelo de regresión logística binaria (Tabla 3), que es donde ingresamos las variables que pudiesen haber influido en la mortalidad de los pacientes, encontramos que únicamente la variable de antecedente de ERC fue un factor de riesgo directo relacionado con la mortalidad (RR 3.73 [IC 95\% 1.28-10.8]; $p=$ 0.01), y dentro de los parámetros del taller gasométrico, encontramos que la $\mathrm{Da}-\mathrm{vO}_{2}$ fue un factor protector en cuanto a mortalidad (RR 0.58 [0.39-0.87]; $p=0.08$ ).

$\mathrm{El}$ índice $\mathrm{Dv}-\mathrm{aCO}_{2} / \mathrm{Da}-\mathrm{vO}_{2}$ no representó una variable de relación directa con la mortalidad, a pesar de que uno de sus determinantes sí lo fue. El poder del modelo generado por la ecuación de regresión

\begin{tabular}{|c|c|}
\hline Variable & $\begin{array}{c}n=136 \\
\text { media } \pm D E\end{array}$ \\
\hline Edad & $66.81 \pm 16.03$ \\
\hline Peso & $68.30 \pm 16.65$ \\
\hline Talla & $1.65 \pm 0.09$ \\
\hline IMC & $25.17 \pm 6.80$ \\
\hline SAPS II & $\begin{array}{c}39.88 \pm 16.09 \\
n=135\end{array}$ \\
\hline APACHE II & $\begin{array}{c}16.69 \pm 7.74 \\
n=135\end{array}$ \\
\hline SOFA ${ }^{*}$ & $\begin{array}{c}8(1-17) \\
n=135\end{array}$ \\
\hline NUTRIC ${ }^{*}$ & $\begin{array}{c}4(1-8) \\
n=135\end{array}$ \\
\hline Diabetes mellitus tipo 2 & $40(29.41)$ \\
\hline Hipertensión & $64(47.06)$ \\
\hline EPOC & $13(9.56)$ \\
\hline Tabaquismo & $72(52.94)$ \\
\hline Cáncer & $60(44.12)$ \\
\hline Cirrosis & $10(7.35)$ \\
\hline ERC & $10(7.35)$ \\
\hline \multicolumn{2}{|l|}{ Motivo de ingreso } \\
\hline No quirúrgico & $102(75.00)$ \\
\hline Quirúrgico & $34(25.00)$ \\
\hline \multicolumn{2}{|l|}{ Sitio de infección } \\
\hline Pulmonar & $58(44.27)$ \\
\hline Gastrointestinal & $34(25.95)$ \\
\hline Nefrourinario & $20(15.27)$ \\
\hline SNC & $5(3.82)$ \\
\hline Piel y tejidos blandos & $3(2.29)$ \\
\hline TSR & $\begin{array}{c}21(15.67) \\
n=134\end{array}$ \\
\hline Días de TSR ${ }^{*}$ & $0(0-30)$ \\
\hline Días de AMV* & $2(0-43)$ \\
\hline Días UCI & $5(1-57)$ \\
\hline Días de estancia hospitalaria* & $11(2-65)$ \\
\hline Muerte & $\begin{array}{c}39(30.00) \\
n=130\end{array}$ \\
\hline
\end{tabular}
utilizado para predecir la mortalidad es analizado en

Tabla 1: Características demográficas basales de los pacientes ingresados con choque séptico.

IMC = índice de masa corporal; EPOC = enfermedad pulmonar obstructiva crónica; $\mathrm{TSR}=$ tratamiento sustitutivo renal; $\mathrm{AMV}=$ asistencia mecánica ventilatoria; $\mathrm{UCl}$ = Unidad de Cuidados Intensivos; * Media \pm DE. 
Tabla 2: Análisis gasométrico de los pacientes sobrevivientes y no sobrevivientes.

\begin{tabular}{|c|c|c|c|}
\hline & Sobrevivientes $n=90$ & Muertos $n=38$ & \\
\hline BE & $-3.2(-15-22)$ & $-2.95(-16-9.5)$ & 0.74 \\
\hline $\mathrm{SvcO}_{2}^{*} \%$ & $70.65 \pm 9.57$ & $71.49 \pm 10.00$ & 0.65 \\
\hline Cortocircuitos, \% & $22.5(4-65)$ & $24(3-63)$ & 0.40 \\
\hline $\mathrm{Dv}-\mathrm{aCO}{ }_{2}, \mathrm{mmHg}$ & $6(1.2-17)$ & $7(2-14)$ & 0.46 \\
\hline Lactato, $\mathrm{mmol} / \mathrm{L}$ & $2.22(0.78-12)$ & $1.9(0.7-14.3)$ & 0.24 \\
\hline Dv-aCO$/ 2 / D a-\mathrm{vO}_{2}$ & $1.73(0.23-9.24)$ & $2.06(0.5-6.66)$ & 0.023 \\
\hline
\end{tabular}

Estadístico de prueba: prueba t de Student; *Prueba de rangos señalados de Wilcoxon; $\mathrm{BE}$ = exceso/déficit de base; $\mathrm{ScvO}_{2}=$ saturación venosa central de oxígeno; IEO $_{2}$ = índice de extracción de oxígeno.

la Figura 1, observando, en la misma figura en A, un AUC de 0.76; esto indica un poder de predicción muy bueno. Además de ello, en la parte $B$, podemos determinar el mejor punto de corte para sensibilidad y especificidad del modelo predictivo generado y su capacidad discriminativa, con lo que se deriva la siguiente ecuación:

Mortalidad $=-1.3100+$ edad $(0.0109)+$ hombre $(0.0825)+$ SOFA $(0.079)+$ ERC $(1.3167)+$ gastrointestinal $(-0.4377)+$ nefrourinario $(0.004)+$ SNC $(-0.1661)+$ gastrointestinal y urinario $(2.0692)+$ pulmonar y SNC (1.2135) + AMV (0.7721) $+\mathrm{DavO}_{2}(-0.5276)$.

\section{DISCUSIÓN}

Hoy en día, la etiología del fallo orgánico es multifactorial, tomando en cuenta que, para que ésta se desarrolle, debe haber alteraciones microcirculatorias, macrocirculatorias, hemodinámicas y metabólicas interrelacionadas. ${ }^{7}$ En los resultados de este estudio, mostramos una estadística de mortalidad similar a otras cohortes internacionales.

La mortalidad en pacientes con choque séptico va de 30 a 37\%, y al existir insuficiencia renal crónica o falla orgánica múltiple, la mortalidad se incrementa hasta $53 \%{ }^{8-10}$ Los resultados obtenidos en este estudio tienen una relación con lo descrito en la literatura mundial,

Tabla 3: Análisis de factores predictores de mortalidad en choque séptico: modelo de regresión logística binaria.

\begin{tabular}{lccc}
\hline Variable & $\mathrm{SE}$ & $\mathrm{RR}(\mathrm{IC} 95 \%)$ & $\mathrm{p}$ \\
\hline ERC & 2.02 & $3.73(1.28-10.8)$ & 0.01 \\
Requerimiento de & 1.25 & $2.16(0.69-6.73)$ & 0.18 \\
ventilación mecánica & & & \\
Da- $\mathrm{vO}_{2}$ & 0.117 & $0.58(0.39-0.87)$ & 0.08 \\
Índice Dv-aCO $/ \mathrm{Da}-\mathrm{vO}_{2}$ & 0.297 & $1.10(0.75-1.61)$ & 0.52 \\
\hline
\end{tabular}

$\mathrm{ERC}=$ antecedente de enfermedad renal crónica; $\mathrm{SE}$ = error estándar; $\mathrm{RR}=$ riesgo relativo. pues encontramos que el antecedente de enfermedad renal crónica en la población evaluada fue un factor de riesgo de mortalidad, cuyo porcentaje va de 42 en comparación con $10 \%$ entre los no sobrevivientes y los sobrevivientes, respectivamente. Al realizar el análisis de regresión, esto fue confirmado como factor de riesgo hasta 2.7 veces más en comparación con aquéllos que no presentaron dicho antecedente (RR 3.73 [IC 95\% 1.28-10.8]; $p=0.01$ ). Asimismo, observamos que los pacientes con deterioro de la función renal aumentaron hasta $100 \%$ el riesgo de requerir terapia de sustitución renal en sus distintas modalidades.

El análisis del taller hemodinámico, al comparar grupos de sobrevivientes y no sobrevivientes al ingreso, mostró un rango de hipoxemia moderada muy similar para ambos grupos $\left(\mathrm{PaO}_{2} / \mathrm{FiO}_{2}\right.$ de 192 vs $175 \mathrm{mmHg}$; $p=0.18)$, sin presentar diferencia estadística significativa. En cuanto a la Dv-aCO$\left(\Delta \mathrm{PCO}_{2}\right)$, encontramos que los pacientes no sobrevivientes presentaron una media de $7 \mathrm{mmHg}$, sin ser un resultado estadísticamente significativo, pero que sí correlaciona con lo descrito en estudios previos como el estudio de Lamsfus, ${ }^{11}$ quien encontró 11 trabajos en la literatura donde se valoraba la $\triangle \mathrm{PCO}_{2}$ como marcador de resucitación en pacientes con sepsis severa y choque séptico, observando que una diferencia $\geq 6 \mathrm{mmHg}$ se asociaba con mayor mortalidad.

Asimismo Mallat y colaboradores ${ }^{12}$ realizaron un estudio prospectivo y observacional, donde 80 pacientes con sepsis severa y choque séptico fueron monitorizados con sistema PiCCO (gasto cardiaco por análisis de contorno de pulso) y sometidos a tratamiento de resucitación habitual, encontrando que una saturación venosa central $\geq 70 \%, \Delta \mathrm{PCO}_{2} \leq 6 \mathrm{mmHg}$ y lactato $\leq 1.7 \mathrm{mmol} / \mathrm{L}$ se asociaban con mayores tasas de supervivencia.

Mesquida y colaboradores ${ }^{13}$ analizaron de manera retrospectiva una muestra de 35 pacientes con diagnóstico de sepsis severa y choque séptico sometidos a tratamiento habitual. Ellos encontraron que los pacientes con una $\triangle \mathrm{PCO}_{2} \geq 6 \mathrm{mmHg}$ tenían mayor mortalidad, 

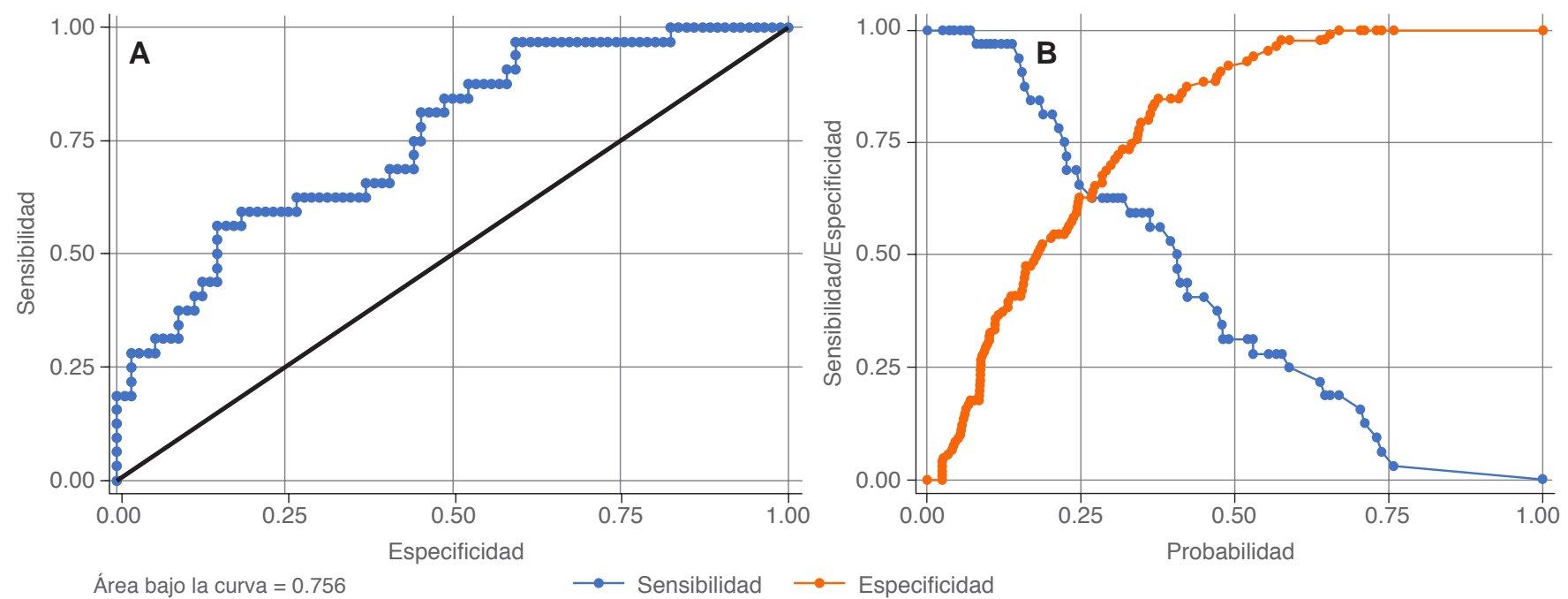

Figura 1: Curvas Receiver Operating Characteristic. Modelo de regresión logística binaria.

la cual se asoció con una depuración de lactato $<10 \%$. Existe también otro estudio realizado en el Centro Médico ABC por la Dra. Hernández y colaboradores, ${ }^{14}$ donde analizaron de manera retrospectiva la $\triangle \mathrm{PCO}_{2}$ en 46 pacientes con choque séptico. En dicho estudio, describieron que la persistencia de una $\triangle \mathrm{PCO}_{2} \geq 6 \mathrm{mmHg}$ posterior a la reanimación resultó en mayor mortalidad.

Encontramos que un perfil hemodinámico sugestivo de hiperdinamia con $\mathrm{Da}-\mathrm{vO}_{2}(2.91 \mathrm{~mL} / \mathrm{dL}$ [1.34-7.7]) fue un determinante directo de mortalidad (RR 0.58 [0.390.87 ]; $p=0.08$ ). Bajo bases fisiológicas, ${ }^{15}$ se describe que el paciente en estado crítico aumenta su respuesta metabólica al estrés, lo que genera mayor demanda de oxígeno por parte de los tejidos y dispara mecanismos de autocompensación, como el aumento del volumen minuto cardiaco. El efecto neto produce una disminución en la diferencia arteriovenosa de oxígeno, ya que se extrae menos oxígeno por cada $\mathrm{dL}$ de sangre que circula, pese a la mayor demanda, y esto va de la mano con la estimulación simpática. ${ }^{16}$

La excesiva estimulación simpática está asociada con efectos adversos a nivel cardiovascular y sistémi$\mathrm{co}$, los cuales pueden afectar negativamente los resultados en el choque séptico. El bloqueo de los receptores $\beta$-adrenérgicos ha mostrado controlar eficazmente el incremento desproporcionado de la frecuencia cardiaca, conservando un perfil hemodinámico favorable $y$, al parecer, mejorando la eficiencia del sistema cardiovascular para mantener la perfusión tisular. Adicionalmente, ha mostrado modular favorablemente la inmunosupresión inducida por catecolaminas y disminuir la resistencia a la insulina, el catabolismo proteico y la expresión de citocinas proinflamatorias asociadas con la disfunción cardiovascular.

Esto ha sido estudiado por varios autores a través de los años. Morelli y colaboradore ${ }^{17}$ encontraron una diferencia estadísticamente significativa al valorar la mortalidad en pacientes que recibieron esmolol en comparación con placebo (49.4 vs $80.5 \%$ ); RR 0.39 e IC $95 \%(0.26-0.59) p<0.001$, respectivamente, sin encontrar un incremento en la presencia de efectos adversos al tratamiento. Sin embargo, estudios posteriores como el de Jacquet-Lagrèze y colaboradore ${ }^{18}$ no demostraron cambios significativos en la mortalidad, únicamente en el control de la frecuencia cardiaca, quienes la consideraron como una opción terapéutica atractiva sin dejarla de lado, considerando los efectos adversos de la respuesta simpática aumentada. Por tal motivo, fue realizado por Vellinga y colaboradores ${ }^{19}$ un ensayo observacional, internacional y multicéntrico en 530 pacientes de cuidados intensivos mixtos, que demostró que la taquicardia y un perfil hiperdinámico era el único parámetro sensible para predecir resultados adversos en los pacientes con choque séptico.

El resto de parámetros del taller gasométrico, tanto en análisis univariado como multivariado, así como la regresión logística binaria para detectar confusores, no presentó resultados con diferencia estadísticamente significativa.

\section{CONCLUSIONES}

El antecedente de enfermedad renal crónica se asocia con una mayor probabilidad de morir. Una diferencia arteriovenosa de oxígeno baja se asocia fuertemente con una mayor probabilidad de morir en pacientes con choque séptico.

El taller gasométrico debe considerarse una herramienta útil, no invasiva e ideal para el análisis de los distintos cambios fisiopatológicos en el paciente con choque séptico. Lo más importante es la interpretación correcta de estas variables, tanto de manera individual 
como en conjunto y su interrelación, pues esto permite un análisis más integral y oportuno para estos pacientes $y$, con ello, se pueden establecer protocolos de tratamientos más razonables sin pérdida de tiempo.

Considerando los efectos deletéreos de la sobreestimulación generada por la actividad simpática, la terapéutica con betabloqueadores puede ser un tratamiento atractivo dirigido en pacientes seleccionados.

\section{BIBLIOGRAFÍA}

1. Angus DC, Linde-Zwirble WT, Lidicker J, Clermont G, Carcillo J, Pinsky MR. Epidemiology of severe sepsis in the United States: analysis of incidence, outcome, and associated costs of care. Crit Care Med. 2001;29(7):1303-1310.

2. Singer M, Deutschman CS, Warren SC, Shankar-Hari M, Annane $\mathrm{D}$, Bauer $\mathrm{M}$, et al. The third international consensus definitions for sepsis and septic shock (sepsis-3). JAMA. 2016;315(8):801810.

3. Shankar-Hari M, Phillips GS, Levy ML, Seymour CW, Liu VX, Deutschman CS, et al. Developing a new definition and assessing new clinical criteria for septic shock: for the Third International Consensus Definitions for sepsis and septic shock (sepsis-3). JAMA. 2016;315(8):775-787.

4. Seymour CW, Liu VX, Iwashyna TJ, Brunkhorst FM, Rea TD, Scherag A, et al. Assessment of clinical criteria for sepsis: for the third international consensus definitions for sepsis and septic shock (sepsis-3). JAMA. 2016;315(8):762-774.

5. Marshall JC, Vincent JL, Guyatt G, Angus DC, Abraham E, Bernard G, Bombardier C, et al. Outcome measures for clinical research in sepsis: a report of the 2 nd Cambridge Colloquium of the International Sepsis Forum. Crit Care Med. 2005;33:17081716.

6. Gattinoni L, Pesenti A, Matthay M. Understanding blood gas analysis. Intensive Care Med. 2018;44(1):91-93.

7. Shoemaker WC, Appel PL, Kram HB, Waxman K, Lee TS. Prospective trial of supranormal values of survivors as therapeutic goals in high-risk surgical patients. Chest. 1988;94:1176-1186.

8. Doi K, Leelahavanichkul A, Hu X, Sidransky KL, Zhou H, Qin Y, et al. Pre-existing renal disease promotes sepsis-induced acute kidney injury and worsens outcome. Kidney Int. 2008;74(8):10171025. doi: 10.1038/ki.2008.346.

9. Dalrymple LS, Go AS. Epidemiology of acute infections among patients with chronic kidney disease. Clin J Am Soc Nephrol. 2008;3(5):1487-1493. doi: 10.2215/CJN.01290308.

10. Naqvi SB, Collins AJ. Infectious complications in chronic kidney disease. Adv Chronic Kidney Dis. 2006;13(3):199-204.
11. Lamsfus-Prieto JÁ, de Castro-Fernández R, Hernández-García AM, Marcano-Rodríguez G: Valor pronóstico de los parámetros gasométricos del dióxido de carbono en pacientes con sepsis. Una revisión bibliográfica. Rev Esp Anestesiol Reanim. 2016;63(4):220-230.

12. Mallat J, Pepy F, Lemyze M, Gasan G, Vangrunderbeeck N, Tronchon $\mathrm{L}$, et al. Central venous to arterial carbon dioxide partial pressure difference in early resuscitation from septic shock. Eur $J$ Anaesthesiol. 2014;31(7):371-380.

13. Mesquida J, Saludes P, Gruartmoner G, Espinal C, Torrents $E$, Baigorri $F$, et al. Central venous to arterial carbon dioxide difference combined with arterial to venous oxygen content difference is associated with lactate evolution in the hemodynamic resuscitation process in early septic shock. Crit Care. 2015;19:1-7.

14. Hernández LA, López PH, Etulain GJ, Olvera GC, Aguirre JS, Franco GJ. Delta de dióxido de carbono para valorar perfusión tisular como predictor de mortalidad en choque séptico. Rev Asoc Mex Med Crit y Ter Int. 2011;25(2):66-70.

15. Annane D, Trabold F, Sharshar T, Jarrin I, Blanc AS, Raphael JC, et al. Inappropriate sympathetic activation at onset of septic shock: a spectral analysis approach. Am J Respir Crit Care Med. 1999;160(2):458-65.

16. Van Loon LM, van der Hoeven JG, Lemson J. Hemodynamic response to $\beta$-blockers in severe sepsis and septic shock: a review of current literature. J Crit Care. 2019;50:138-143. doi: 10.1016/j.jcrc.2018.12.003.

17. Morelli A, Ertmer C, Westphal M, Rehberg S, Kampmeier $\mathrm{T}$, Ligges $\mathrm{S}$, et al. Effect of heart rate control with esmolol on hemodynamic and clinical outcomes in patients with septic shock: a randomized clinical trial. JAMA. 2013;310:1683-1691.

18. Jacquet-Lagrèze $M$, Allaouchiche $B$, Restagno $D$, Paquet $C$, Ayoub JY, Etienne J, et al. Gut and sublingual microvascular effect of esmolol during septic shock in a porcine model. Crit Care. 2015;4:19-241.

19. Vellinga NA, Boerma EC, Koopmans M, Donati A, Dubin A, Shapiro NI, et al. International study on microcirculatory shock occurrence in acutely ill patients. Crit Care Med. 2015;43:48-56.

\section{Conflicto de intereses: Ninguno.}

\section{Correspondencia:}

Dr. Alfredo Aisa-Álvarez

Avenida Carlos Graef Fernández Núm. 154,

Col. Santa Fe, 05300,

Alcaldía Cuajimalpa de Morelos,

Ciudad de México, México.

E-mail: alfredoaisaa@gmail.com 